\title{
A Scale-Space Approach to Nonlocal Optical Flow Calculations
}

\author{
Luis Alvarez ${ }^{1}$, Joachim Weickert $^{2}$, and Javier Sánchez ${ }^{1}$ \\ ${ }^{1}$ Departamento de Informática y Sistemas, \\ Universidad de Las Palmas, Campus de Tafira, \\ SP-35017 Las Palmas, Spain. \\ E-Mail: $\{$ lalvarez, jsanchez\}@dis.ulpgc.es \\ WWW: http://serdis.dis.ulpgc.es/ \{lalvarez,jsanchez $\}$ \\ 2 Computer Vision, Graphics, and Pattern Recognition Group, \\ Department of Mathematics and Computer Science, \\ University of Mannheim, D-68131 Mannheim, Germany. \\ E-Mail: Joachim.Weickert@ti.uni-mannheim.de \\ WWW: http://www.ti.uni-mannheim.de/ bmg/weickert
}

\begin{abstract}
This paper presents an interpretation of a classic optical flow method by Nagel and Enkelmann as a tensor-driven anisotropic diffusion approach in digital image analysis. We introduce an improvement into the model formulation, and we establish well-posedness results for the resulting system of parabolic partial differential equations. Our method avoids linearizations in the optical flow constraint, and it can recover displacement fields which are far beyond the typical one-pixel limits that are characteristic for many differential methods for optical flow recovery. A robust numerical scheme is presented in detail. We avoid convergence to irrelevant local minima by embedding our method into a linear scalespace framework and using a focusing strategy from coarse to fine scales. The high accuracy of the proposed method is demonstrated by means of a synthetic and a real-world image sequence.
\end{abstract}

\section{Introduction}

Optical flow computation consists of finding the apparent motion of objects in a sequence of images. It is a key problem in artificial vision and much research has been devoted to this field; for a survey see e.g. [23].

In the present paper we shall consider two images $I_{1}(x, y)$ and $I_{2}(x, y)$ (defined on $\mathbb{R}^{2}$ to simplify the discussion) which represent two consecutive views in a sequence of images. Under the assumption that corresponding pixels have equal grey values, the determination of the optical flow from $I_{1}$ to $I_{2}$ comes down to finding a function $h(x, y)=(u(x, y), v(x, y))$ such that

$$
I_{1}(x, y)=I_{2}(x+u(x, y), y+v(x, y)), \quad \forall(x, y) \in \mathbb{R}^{2} .
$$

To compute $h(x, y)$ the preceding equality is usually linearized yielding the so-called optical flow constraint

$$
I_{1}(\bar{x})-I_{2}(\bar{x}) \approx\left\langle\nabla I_{2}(\bar{x}), h(\bar{x})\right\rangle \quad \forall \bar{x} \in \mathbb{R}^{2},
$$


where $\bar{x}:=(x, y)$. The linearized optical flow constraint assumes that the object displacements $h(\bar{x})$ are small or that the image is slowly varying in space. In other cases, this linearization is no longer valid.

Frequently, instead of equation (1), researchers use the alternative equality

$$
I_{1}(x-u(x, y), y-v(x, y))=I_{2}(x, y), \quad \forall(x, y) \in \mathbb{R}^{2} .
$$

In this case the displacement $h(x, y)$ is centered in the image $I_{2}(x, y)$.

The determination of optical flow is a classic ill-posed problem in computer vision [7], and it requires to be supplemented with additional regularizing assumptions. The regularization by Horn and Schunck [16] assumes that the optical flow field is smooth. However, since many natural image sequences are better described in terms of piecewise smooth flow fields separated by discontinuities, much research has been done to modify the Horn and Schunck approach in order to permit such discontinuous flow fields; see e.g. [8, 10, 11, 21, 24, 25, 27,32] and the references therein.

An important improvement in this direction has been achieved by Nagel and Enkelmann [24] in 1986. They consider the following minimization problem:

$$
\begin{aligned}
E_{N E}(h)= & \int_{\mathbb{R}^{2}}\left(I_{1}(x-u(x, y), y-v(x, y))-I_{2}(x, y)\right)^{2} d x \\
& +C \int_{\mathbb{R}^{2}} \operatorname{trace}\left((\nabla h)^{T} D\left(\nabla I_{1}\right)(\nabla h)\right) d x
\end{aligned}
$$

where $C$ is a positive constant and $D\left(\nabla I_{1}\right)$ is a regularized projection matrix in the direction perpendicular of $\nabla I_{1}$ :

$$
D\left(\nabla I_{1}\right)=\frac{1}{\left|\nabla I_{1}\right|^{2}+2 \lambda^{2}}\left\{\left(\begin{array}{c}
\frac{\partial I_{1}}{\partial y} \\
-\frac{\partial I_{1}}{\partial x}
\end{array}\right)\left(\begin{array}{c}
\frac{\partial I_{1}}{\partial y} \\
-\frac{\partial I_{1}}{\partial x}
\end{array}\right)^{T}+\lambda^{2} I d\right\} .
$$

In this formulation, $I d$ denotes the identity matrix. The advantage of this method is that it inhibits blurring of the flow across boundaries of $I_{1}$ where $\left|\nabla I_{1}\right|>>\lambda$. This model, however, uses an optical flow constraint which is centered in $I_{2}$, while the projection matrix $D$ in the smoothness term depends on $I_{1}$. This inconsistency may lead to erroneous results for large displacement fields. In order to avoid this problem, we consider a modified energy functional where both the optical flow constraint and the smoothness constraint are related to $I_{1}$ :

$$
\begin{aligned}
E(h)= & \int_{\mathbb{R}^{2}}\left(I_{1}(x, y)-I_{2}(x+u(x, y), y+v(x, y))\right)^{2} d x \\
& +C \int_{\mathbb{R}^{2}} \operatorname{trace}\left((\nabla h)^{T} D\left(\nabla I_{1}\right)(\nabla h)\right) d x .
\end{aligned}
$$


The associated Euler-Lagrange equations are given by the PDE system

$$
\begin{aligned}
& C \operatorname{div}\left(D\left(\nabla I_{1}\right) \nabla u\right)+\left(I_{1}(\bar{x})-I_{2}(\bar{x}+h(\bar{x}))\right) \frac{\partial I_{2}}{\partial x}(\bar{x}+h(\bar{x}))=0 \\
& C \operatorname{div}\left(D\left(\nabla I_{1}\right) \nabla v\right)+\left(I_{1}(\bar{x})-I_{2}(\bar{x}+h(\bar{x}))\right) \frac{\partial I_{2}}{\partial y}(\bar{x}+h(\bar{x}))=0 .
\end{aligned}
$$

In this paper, we are interested in solutions of the equations (5)-(6) in the case of large displacement fields and images that are not necessarily slowly varying in space. Therefore, we do not introduce any linearization in the above system. We obtain the solutions by calculating the asymptotic state $(t \rightarrow \infty)$ of the parabolic system

$$
\begin{aligned}
& \frac{\partial u}{\partial t}=C \operatorname{div}\left(D\left(\nabla I_{1}\right) \nabla u\right)+\left(I_{1}(\bar{x})-I_{2}(\bar{x}+h(\bar{x}))\right) \frac{\partial I_{2}}{\partial x}(\bar{x}+h(\bar{x})), \\
& \frac{\partial v}{\partial t}=C \operatorname{div}\left(D\left(\nabla I_{1}\right) \nabla v\right)+\left(I_{1}(\bar{x})-I_{2}(\bar{x}+h(\bar{x}))\right) \frac{\partial I_{2}}{\partial y}(\bar{x}+h(\bar{x})) .
\end{aligned}
$$

Interestingly, this coupled system of diffusion-reaction equations reveals a diffusion tensor which resembles the one used for edge-enhancing anisotropic diffusion filtering. Indeed, $D\left(\nabla I_{1}\right)$ has the eigenvectors $v_{1}:=\nabla I_{1}$ and $v_{2}:=$ $\nabla I_{1}^{\perp}$. The corresponding eigenvalues are given by

$$
\begin{aligned}
& \lambda_{1}\left(\left|\nabla I_{1}\right|\right)=\frac{\lambda^{2}}{\left|\nabla I_{1}\right|^{2}+2 \lambda^{2}}, \\
& \lambda_{2}\left(\left|\nabla I_{1}\right|\right)=\frac{\left|\nabla I_{1}\right|^{2}+\lambda^{2}}{\left|\nabla I_{1}\right|^{2}+2 \lambda^{2}} .
\end{aligned}
$$

We observe, that $\lambda_{1}+\lambda_{2}=1$ holds independently of $\nabla I_{1}$. In the interior of objects we have $\left|\nabla I_{1}\right| \rightarrow 0$, and therefore $\lambda_{1} \rightarrow 1 / 2$ and $\lambda_{2} \rightarrow 1 / 2$. At ideal edges where $\left|\nabla I_{1}\right| \rightarrow \infty$, we obtain $\lambda_{1} \rightarrow 0$ and $\lambda_{2} \rightarrow 1$. Thus, we have isotropic behaviour within regions, and at image boundaries the process smoothes anisotropically along the edge. This behaviour is very similar to edgeenhancing anisotropic diffusion filtering [30], and it is also close in spirit to the modified mean-curvature motion considered in [3]. In this sense, one may regard the Nagel-Enkelmann method as an early predecessor of modern PDE techniques for image restoration. For a detailed treatment of anisotropic diffusion filtering we refer to [31], and an axiomatic classification of mean-curvature motion and related morphological PDEs for image analysis is presented in [2].

Without any linearization, the optical flow constraint may cause a nonconvex energy functional (4). In this case we cannot expect the uniqueness of solutions of the elliptic system (5)-(6), and the asymptotic state of the above parabolic system depends on the initial data for the flow $u$ and $v$. In order to encourage convergence to the physically correct solution in case of large displacement flow, we will design a linear scale-space focusing procedure for the optical flow constraint. Using a scale-space approach enables us also to perform a finer and 
more reliable scale focusing as it would be the case for related pyramid [4] or multigrid approaches [12].

The paper is organized as follows: In Section 2 we sketch existence and uniqueness results for the nonlinear parabolic system (7)-(8). In Section 3 we apply a linear scale-space focusing which enables us to achieve convergence to realistic solutions for large displacement vectors. Section 4 describes a numerical discretization of the parabolic system (7)-(8) based on an explicit finite difference scheme. In Section 5 we present experimental results on a synthetic and a real-world image sequence. Finally, in Section 6 we conclude with a summary.

Related work. Proesmans et al. [25] studied a related approach that also dispenses with a linearization of the optical flow constraint in order to allow for larger displacements. Their method, however, requires six coupled partial differential equations and its nonlinear diffusion process uses a scalar-valued diffusivity instead of a diffusion tensor. Their discontinuity-preserving smoothing is flow-driven while ours is image-driven. Another PDE technique that is similar in vein to the work of Proesmans et al. is a stereo method due to Shah [28]. With respect to embeddings into a linear scale-space framework our method can be related to the optical flow approach of Florack et al. [14]. Their method differs from ours in that it is purely linear, applies scale selection mechanisms and does not use discontinuity-preserving nonlinear smoothness terms. Our focusing strategy for avoiding to end up in irrelevant local minima also resembles the graduated non-convexity (GNC) algorithms of Blake and Zisserman [9].

\section{Existence and Uniqueness of the Parabolic System}

Next we investigate the parabolic system of nonlinear partial differential equations (7)-(8). In [1], the authors develop a theoretical framework to study the existence and uniqueness of solutions of a similar parabolic system, but with a different regularization term. The main techniques used in [1] can be applied in order to obtain the existence and uniqueness of the solutions of the system (7)-(8). This leads to the following result.

Theorem 1. Let $I_{2} \in C^{2}\left(\mathbb{R}^{2}\right)$ and $I_{1} \in C^{1}\left(\mathbb{R}^{2}\right)$. Then the parabolic system (7)(8) has a unique generalized solution $h(., t) \in C\left([0, \infty) ; L^{2}\left(\mathbb{R}^{2}\right) \times L^{2}\left(\mathbb{R}^{2}\right)\right)$ for all initial flows $h_{0} \in L^{2}\left(\mathbb{R}^{2}\right) \times L^{2}\left(\mathbb{R}^{2}\right)$.

\section{A Linear Scale-Space Approach to Recover Large Displacements}

In general, the Euler-Lagrange equations (5)-(6) will have multiple solutions. As a consequence, the asymptotic state of the parabolic system (7)-(8), which we use for approximating the optical flow, will depend on the initial data. Typically, the convergence is the better, the closer the initial data is to the asymptotic state. When we expect small displacements in the scene, the natural choice is to take 
$u \equiv v \equiv 0$ as initialization of the flow. For large displacement fields, however, this may not work, and we need better initial data. To this end, we embed our method into a linear scale-space framework $[17,33]$. Considering the problem at a coarse scale avoids that the algorithm gets trapped in physically irrelevant local minima. The coarse-scale solution serves then as initial data for solving the problem at a finer scale. Scale focusing has a long tradition in linear scale-space theory (see e.g. Bergholm [6] for an early approach), and in spite of the fact that several theoretical problems exist, it has not lost its popularity due to its favourable practical behaviour. Detailed descriptions of linear scale-space theory can be found in $[13,15,18,19,22,29]$.

We proceed as follows. First, we introduce a linear scale factor in the parabolic PDE system in order to end up with

$$
\begin{aligned}
\frac{\partial u_{\sigma}}{\partial t} & =C \operatorname{div}\left(D\left(\nabla G_{\sigma} * I_{1}\right) \nabla u_{\sigma}\right)+ \\
& +\left(G_{\sigma} * I_{1}(\bar{x})-G_{\sigma} * I_{2}\left(\bar{x}+h_{\sigma}(\bar{x})\right)\right) \frac{\partial\left(G_{\sigma} * I_{2}\right)}{\partial x}\left(\bar{x}+h_{\sigma}(\bar{x})\right), \\
\frac{\partial v_{\sigma}}{\partial t} & =C \operatorname{div}\left(D\left(\nabla G_{\sigma} * I_{1}\right) \nabla v_{\sigma}\right)+ \\
& +\left(G_{\sigma} * I_{1}(\bar{x})-G_{\sigma} * I_{2}\left(\bar{x}+h_{\sigma}(\bar{x})\right)\right) \frac{\partial\left(G_{\sigma} * I_{2}\right)}{\partial y}\left(\bar{x}+h_{\sigma}(\bar{x})\right),
\end{aligned}
$$

where $G_{\sigma} * I$ represents the convolution of $I$ with a Gaussian of standard deviation $\sigma$.

The convolution with a Gaussian blends the information in the images and allows us to recover a connection between the objects in $I_{1}$ and $I_{2}$. We start with a large initial scale $\sigma_{0}$. Then we compute the optical flow $\left(u_{\sigma_{0}}, v_{\sigma_{0}}\right)$ at scale $\sigma_{0}$ as the asymptotic state of the solution of the above PDE system using as initial data $u \equiv v \equiv 0$. Next, we choose a number of scales $\sigma_{n}<\sigma_{n-1}<\ldots<\sigma_{0}$, and for each scale $\sigma_{i}$ we compute the optical flow $\left(u_{\sigma_{i}}, v_{\sigma_{i}}\right)$ as the asymptotic state of the above PDE system with initial data $\left(u_{\sigma_{i-1}}, v_{\sigma_{i-1}}\right)$. The final computed flow corresponds to the smallest scale $\sigma_{n}$. In accordance with the logarithmic sampling strategy in linear scale-space theory [20], we choose $\sigma_{i}:=\eta^{i} \sigma_{0}$ with some decay rate $\eta \in(0,1)$.

\section{Numerical Scheme}

We discretize the parabolic system (11)-(12) by finite differences. All spatial derivatives are approximated by central differences, and for the discretization in $t$ direction we use an explicit (Euler forward) scheme. Gaussian convolution was performed in the spatial domain with renormalized Gaussians, which where truncated at 5 times their standard deviation. Let $D\left(\nabla G_{\sigma} * I_{1}\right)=\left(\begin{array}{ll}a & b \\ b & c\end{array}\right)$. Then 
our explicit scheme has the structure

$$
\begin{aligned}
\frac{u_{i, j}^{k+1}-u_{i, j}^{k}}{\tau} & =C\left(\frac{a_{i+1, j}+a_{i, j}}{2} \frac{u_{i+1, j}^{k}-u_{i, j}^{k}}{h_{1}^{2}}+\frac{a_{i-1, j}+a_{i, j}}{2} \frac{u_{i-1, j}^{k}-u_{i, j}^{k}}{h_{1}^{2}}+\right. \\
& +\frac{c_{i, j+1}+c_{i, j}}{2} \frac{u_{i, j+1}^{k}-u_{i, j}^{k}}{h_{2}^{2}}+\frac{c_{i, j-1}+c_{i, j}}{2} \frac{u_{i, j-1}^{k}-u_{i, j}^{k}}{h_{2}^{2}}+ \\
& +\frac{b_{i+1, j+1}+b_{i, j}}{2} \frac{u_{i+1, j+1}^{k}-u_{i, j}^{k}}{2 h_{1} h_{2}}+\frac{b_{i-1, j-1}+b_{i, j}}{2} \frac{u_{i-1, j-1}^{k}-u_{i, j}^{k}}{2 h_{1} h_{2}}- \\
& \left.-\frac{b_{i+1, j-1}+b_{i, j}}{2} \frac{u_{i+1, j-1}^{k}-u_{i, j}^{k}}{2 h_{1} h_{2}}-\frac{b_{i-1, j+1}+b_{i, j}}{2} \frac{u_{i-1, j+1}^{k}-u_{i, j}^{k}}{2 h_{1} h_{2}}\right)+ \\
& +\left(I_{1, \sigma}\left(\bar{x}_{i, j}^{k}\right)-I_{2, \sigma}\left(\bar{x}_{i, j}+\bar{h}_{\sigma, i, j}^{k}\right)\right) I_{2, x, \sigma}\left(\bar{x}_{i, j}+\bar{h}_{\sigma, i, j}^{k}\right), \\
\frac{v_{i, j}^{k+1}-v_{i, j}^{k}}{\tau} & =C\left(\frac{a_{i+1, j}+a_{i, j}}{2} \frac{v_{i+1, j}^{k}-v_{i, j}^{k}}{h_{1}^{2}}+\frac{a_{i-1, j}+a_{i, j}}{2} \frac{v_{i-1, j}^{k}-v_{i, j}^{k}}{h_{1}^{2}}+\right. \\
& +\frac{c_{i, j+1}+c_{i, j}}{2} \frac{v_{i, j+1}^{k}-v_{i, j}^{k}}{h_{2}^{2}}+\frac{c_{i, j-1}+c_{i, j}}{2} \frac{v_{i, j-1}^{k}-v_{i, j}^{k}}{h_{2}^{2}}+ \\
& +\frac{b_{i+1, j+1}+b_{i, j}}{2} \frac{v_{i+1, j+1}^{k}-v_{i, j}^{k}}{2 h_{1} h_{2}}+\frac{b_{i-1, j-1}+b_{i, j}}{2} \frac{v_{i-1, j-1}^{k}-v_{i, j}^{k}}{2 h_{1} h_{2}}- \\
& \left.-\frac{b_{i+1, j-1}+b_{i, j}}{2} \frac{v_{i+1, j-1}^{k}-v_{i, j}^{k}}{2 h_{1} h_{2}}-\frac{b_{i-1, j+1}+b_{i, j}}{2} \frac{v_{i-1, j+1}^{k}-v_{i, j}^{k}}{2 h_{1} h_{2}}\right)+ \\
& +\left(I_{1, \sigma}\left(\bar{x}_{i, j}^{k}\right)-I_{2, \sigma}\left(\bar{x}_{i, j}+\bar{h}_{\sigma, i, j}^{k}\right)\right) I_{2, y, \sigma}\left(\bar{x}_{i, j}+\bar{h}_{\sigma, i, j}^{k}\right) .
\end{aligned}
$$

The notations are almost selfexplaining: for instance, $\tau$ is the time step size, $h_{1}$ and $h_{2}$ denote the pixel size in $x$ and $y$ direction, respectively, $u_{i, j}^{k}$ approximates $u_{\sigma}$ in some grid point $\bar{x}_{i, j}$ at time $k \tau$, and $I_{1, x, \sigma}$ is an approximation to $G_{\sigma} * \frac{\partial I_{1}}{\partial x}$. We calculate values of type $I_{2, \sigma}\left(\bar{x}_{i, j}+\bar{h}_{\sigma, i, j}^{k}\right)$ by linear interpolation, and we use the time step size

$$
\tau=\frac{0.5}{4 C+\max _{i, j}\left(\left|I_{1, x, \sigma}\left(\bar{x}_{i, j}\right)\right|^{2},\left|I_{1, y, \sigma}\left(\bar{x}_{i, j}\right)\right|^{2}\right)} .
$$

This step size can be motivated from a stability analysis in the maximum norm applied to a simplification of (13)-(14) where a scalar-valued diffusivity and a linearized optical flow constraint is used.

\section{$5 \quad$ Experimental Results}

The complete algorithm for computing the optical flow depends on a number of parameters which have an intuitive meaning: 
- The regularization parameter $C$ specifies the balance between the smoothing term and the optical flow constraint. Larger values lead to smoother flow fields by filling in information from image edges where flow measurements with higher reliability are available. Recent results show that there is also a close relationship between the parameter $C$ of a regularization method and the scale parameter of a diffusion scale-space [26].

- The constant $\lambda$ in the smoothing term serves as a contrast parameter: locations where the image gradient magnitude is larger than $\lambda$ are regarded as edges. The diffusion process smoothes anisotropically along these edges. In our experiments we used $\lambda:=1$. The results were not very sensitive to underestimations of $\lambda$.

- The scale $\sigma_{0}$ denotes the standard deviation of the largest Gaussian. In general, $\sigma_{0}$ is chosen according to the maximum displacement expected. In our case we used $\sigma_{0}:=10$.

- The decay rate $\eta \in(0,1)$ for the computation of the scales $\sigma_{m}:=\eta^{m} \sigma_{0}$. We may expect a good focusing if $\eta$ is close to 1 . We have chosen $\eta:=0.95$.

- The smallest scale is given by $\sigma_{n}$. It should be close to the inner scale of the image in order to achieve optimal flow localization.

- The stopping time $T$ for solving the system (11)-(12) at each scale $\sigma_{m}$. When good initializations from coarser scales are available, we observed that $T:=20$ gives results which are sufficiently close to the asymptotic state.

Figure 1 shows our first experiment. We use a synthetic image composed of four black squares on a white background. Each square moves in a different direction and with a different displacement magnitude: under the assumption that the $x$ axis is oriented from left to right and the $y$ axis from top to bottom, the left square on the top moves with $(u, v)=(10,5)$, the right square on the top is displaced with $(u, v)=(-10,0)$, the left square on the bottom is shifted by $(u, v)=(0,-5)$, and the right square on the bottom undergoes a translation by $(-10,-10)$. In order to visualize the flow field $(u, v)$ we use two grey level images $\left(u_{g l}, v_{g l}\right)$ defined by $u_{g l}:=128+8 u$ and $v_{g l}:=128+8 v$. We use the regularization parameter $C=15000$. The depicted optical flow was obtained without scale-space focusing, i.e. with $\sigma_{0}=0$. As can be expected, the algorithm gets trapped in a physically irrelevant local minimum.

Figure 2 shows that the proposed scale-space focusing leads to significantly improved results. We start with initial scale $\sigma_{0}=10$ and show the results for focusing to the scales $\sigma_{10}=5.99, \sigma_{20}=3.58, \sigma_{30}=2.15, \sigma_{40}=1.29, \sigma_{50}=0.77$, $\sigma_{60}=0.46$, and $\sigma_{70}=0.28$, respectively. The other parameters are identical with those in Figure 1. We notice that the computed flow is a good approximation of the expected flow. In fact, not only the orientation of the flow is correct, but also the flow magnitude is surprisingly accurate: the maximum of the computed optic flow magnitude is 14.13 , which is a very good approximation of the ground truth maximum $10 \sqrt{2} \approx 14.14$. It results from the square which moves in $(-10,-10)$ direction. This indicates that - under specific circumstances - our method may even lead to optical flow results with subpixel accuracy.

This observation is confirmed in the quantitative evaluations carried out in Figure 3 . The left plot shows the average angular errors in the four squares of the 


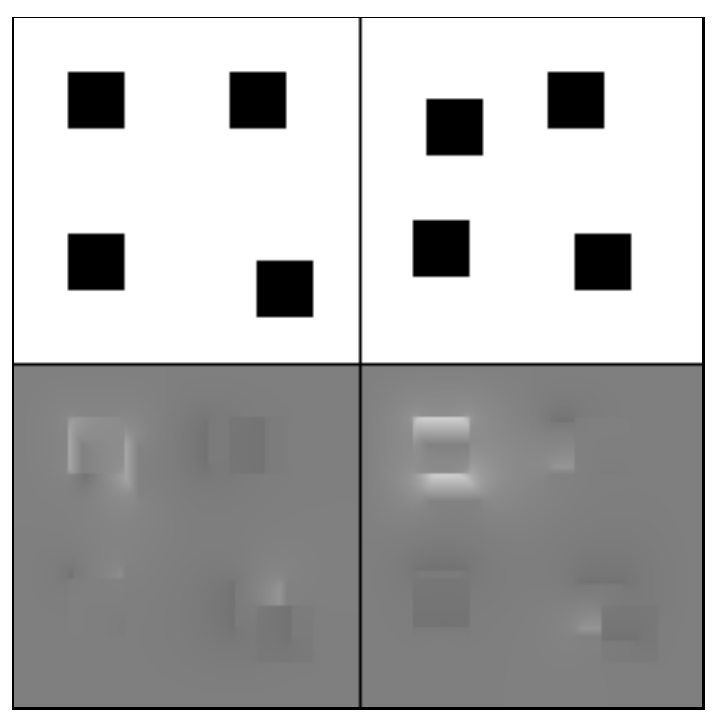

Fig. 1. Optic flow obtained without scale-space focusing $(T=800)$.

first frame. The angels between the correct flow $\left(u_{c}, v_{c}\right)$ and the estimated flow $\left(u_{e}, v_{e}\right)$ have been calculated in the same way as in [5]. The right plot depicts the Euclidean error $\sqrt{\left(u_{e}-u_{c}\right)^{2}+\left(v_{e}-v_{c}\right)^{2}}$ averaged over all pixels within the four squares of the first frame. In both cases we observe that the error is reduced dramatically by focusing down in scale-space until it reaches a very small value when the Gaussian width $\sigma$ approaches the inner scale of the image. Further reductions of $\sigma$ leads to slightly higher errors. It appears that this is caused by discretization effects.

In the fourth experiment, we use the classical taxi sequence, but instead of taking two consecutive frames - as is usually done - we consider the frames 15 and 19. The dark car at the left creates a largest displacement magnitude of approximately 12 pixels. In Figure 4 we present the computed flow using the regularization parameter $C=500$ and focusing from $\sigma_{0}=10$ to $\sigma_{70}=0.28$. The computed maximal flow magnitude is 11.68 , which is a good approximation of the actual displacement of the dark car. Figure 5 shows a vector plot of the computed flow field.

\section{Conclusions}

Usually, when computer vision researchers deal with variational methods for optical flow calculations, they linearize the optical flow constraint. Except for those cases where the images a sufficiently slowly varying in space, linearization, however, does only work for small displacements. In this paper we investigate 


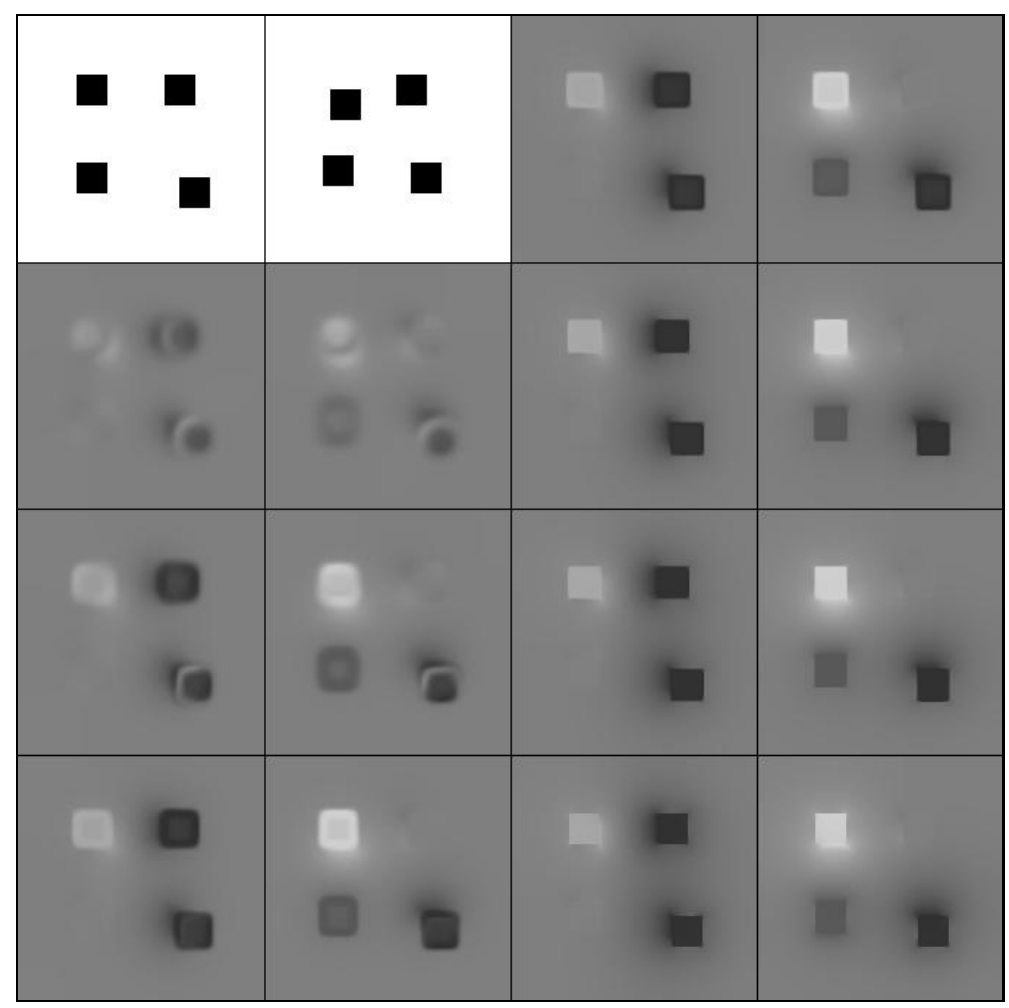

Fig. 2. From top to bottom and from left to right: the original pair of images $I_{1}$ and $I_{2}$, and the flow components $\left(u_{n}, v_{n}\right)$ resulting from focusing to the scales $\sigma_{10}=5.99, \sigma_{20}=3.58, \sigma_{30}=2.15, \sigma_{40}=1.29$, $\sigma_{50}=0.77, \sigma_{60}=0.46$, and $\sigma_{70}=0.28$, respectively.
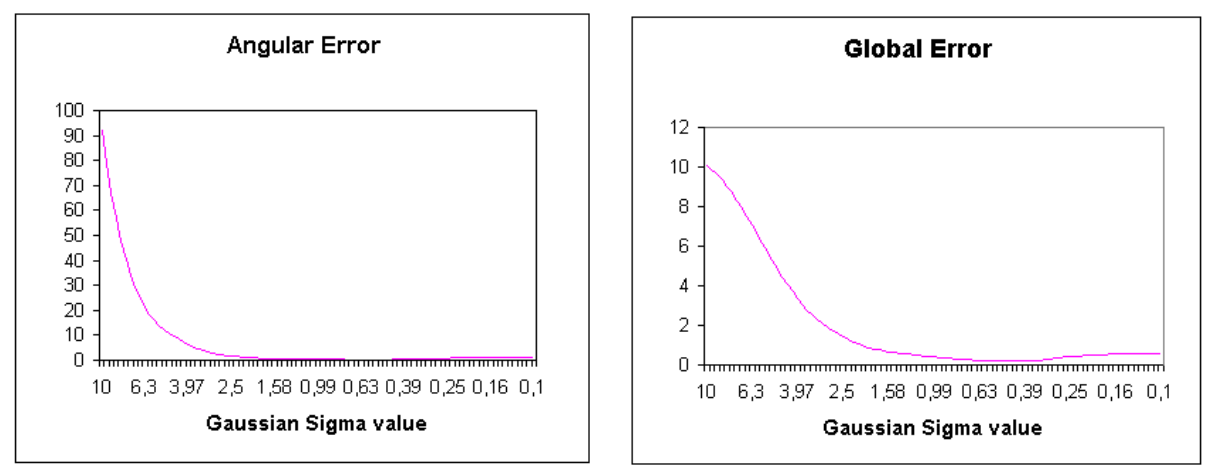

Fig. 3. Left: Average angular error of the optic flow calculations for the squares in the first frame of Figure 2. Right: Corresponding average Euclidean error. 


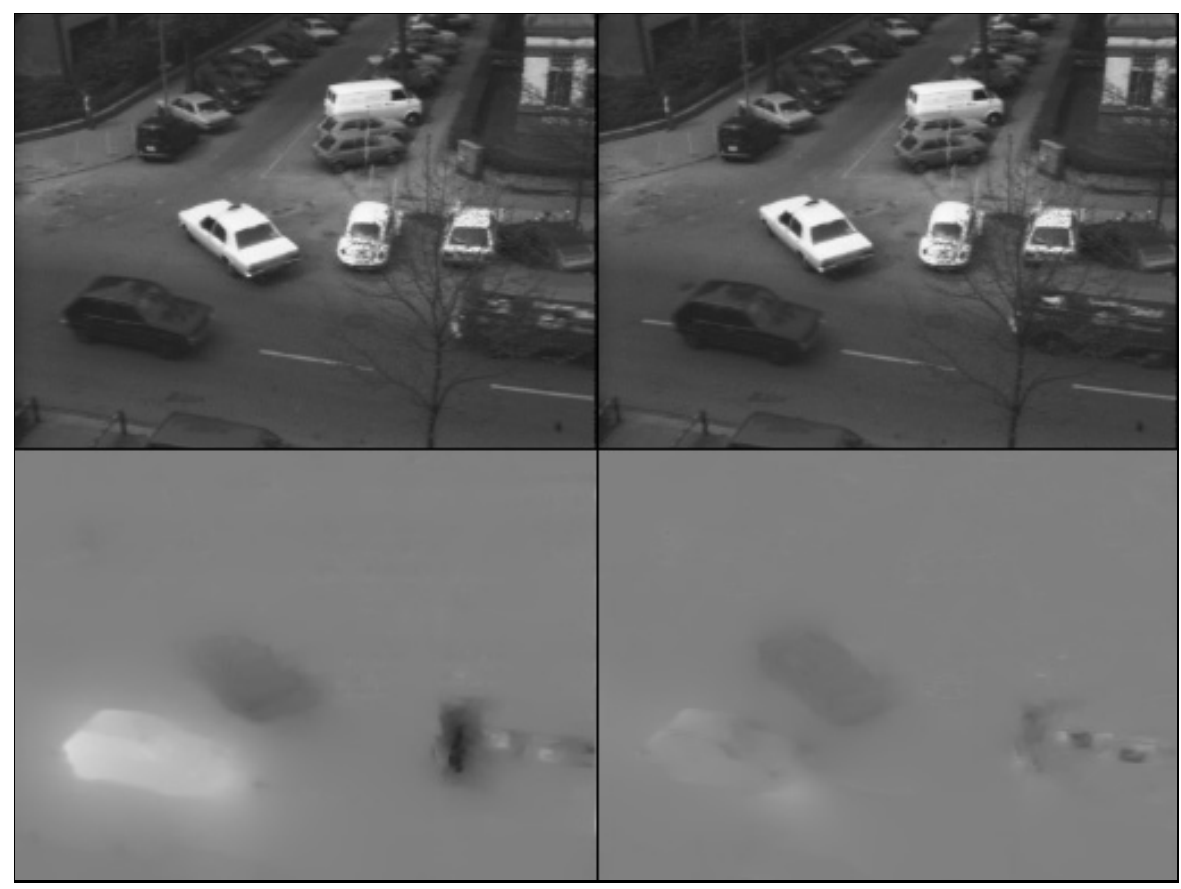

Fig. 4. Optic flow computation of the taxi sequence using frames 15 and 19 .

an improved formulation of a classical method by Nagel and Enkelmann where no linearization is used. We identify this method with two coupled anisotropic diffusion filters with a nonlinear reaction term. We showed that this parabolic system is well-posed from a mathematical viewpoint, and we presented a finite difference scheme for its numerical solution. In order to avoid that the algorithms converges to physically irrelevant local minima, we embedded it into a linear scale-space approach for focusing the solution from a coarse to a fine scale. The numerical results that we have presented for a synthetic and a real-world sequence are very encouraging: it was possible to recover displacements of more than 10 pixels with high accuracy. It is our hope that this successful blend of nonlinear anisotropic PDEs and linear scale-space techniques may serve as a motivation to study other combinations of linear and nonlinear scale-space approaches in the future.

Acknowledgements. This work has been supported by the European TMR network Viscosity Solutions and their Applications.

\section{References}

1. L. Alvarez, J. Esclarín, M. Lefébure and J. Sánchez, A PDE model for computing the optical flow, Proc. XVI Congreso de Ecuaciones Diferenciales y Aplicaciones 


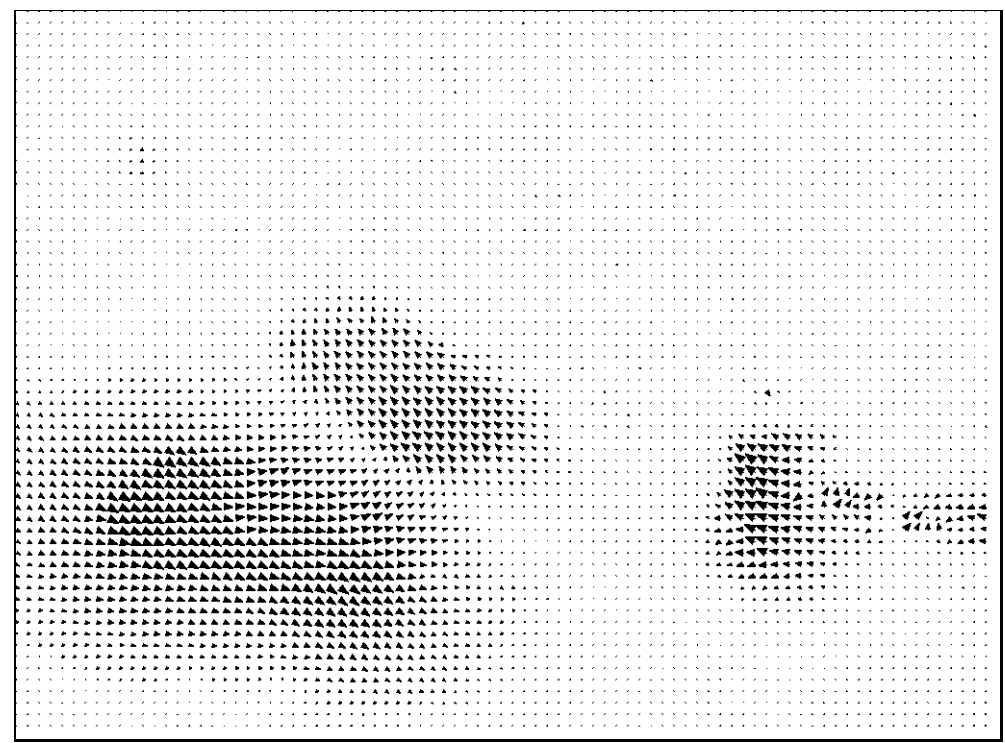

Fig. 5. Vector plot of the optic flow between the frames 15 and 19 of the taxi sequence.

(C.E.D.Y.A. XVI, Las Palmas de Gran Canaria, Sept. 21-24, 1999), in press.

2. L. Alvarez, F. Guichard, P.-L. Lions, J.-M. Morel, Axioms and fundamental equations in image processing, Arch. Rational Mech. Anal., Vol. 123, 199-257, 1993.

3. L. Alvarez, P.-L. Lions, J.-M. Morel, Image selective smoothing and edge detection by nonlinear diffusion. II, SIAM J. Numer. Anal., Vol. 29, 845-866, 1992.

4. P. Anandan, A computational framework and an algorithm for the measurement of visual motion, Int. J. Comput. Vision, Vol. 2, 283-310, 1989.

5. J.L. Barron, D.J. Fleet, S.S. Beauchemin, Performance of optical flow techniques, Int. J. Comput. Vision, Vol. 12, 43-77, 1994.

6. F. Bergholm, Edge focusing, IEEE Trans. Pattern Anal. Mach. Intell., Vol. 9, 726741, 1987.

7. M. Bertero, T.A. Poggio, V. Torre, Ill-posed problems in early vision, Proc. IEEE, Vol. 76, 869-889, 1988.

8. M.J. Black, P. Anandan, Robust dynamic motion estimation over time, Proc. IEEE Comp. Soc. Conf. on Computer Vision and Pattern Recognition (CVPR '91, Maui, June 3-6, 1991), IEEE Computer Society Press, Los Alamitos, 292-302, 1991.

9. A. Blake, A. Zisserman, Visual reconstruction, MIT Press, Cambridge (Mass.), 1987.

10. I. Cohen, Nonlinear variational method for optical flow computation, Proc. Eighth Scandinavian Conf. on Image Analysis (SCIA '93, Tromsø, May 25-28, 1993), Vol. 1, 523-530, 1993.

11. R. Deriche, P. Kornprobst, G. Aubert, Optical-flow estimation while preserving its discontinuities: A variational approach, Proc. Second Asian Conf. Computer Vision (ACCV '95, Singapore, December 5-8, 1995), Vol. 2, 290-295, 1995. 
12. W. Enkelmann, Investigation of multigrid algorithms for the estimation of optical flow fields in image sequences, Computer Vision, Graphics and Image Processing, Vol. 43, 150-177, 1988.

13. L. Florack, Image structure, Kluwer, Dordrecht, 1997.

14. L.M.J. Florack, W.J. Niessen, M. Nielsen, The intrinsic structure of the optic flow incorporating measurement duality, Int. J. Comput. Vision, Vol. 27, 263-286, 1998.

15. B. ter Haar Romeny, L. Florack, J. Koenderink, M. Viergever (Eds.), Scale-space theory in computer vision, Lecture Notes in Computer Science, Vol. 1252, Springer, Berlin, 1997.

16. B. Horn, B. Schunck, Determining optical flow, Artif. Intell., Vol. 17, 185-203, 1981.

17. T. Iijima, Basic theory on normalization of pattern (in case of typical onedimensional pattern), Bulletin of the Electrotechnical Laboratory, Vol. 26, 368-388, 1962 (in Japanese).

18. T. Iijima, Pattern recognition, Corona-sha, 1973 (in Japanese).

19. T. Iijima, Theory of pattern recognition, Series of Basic Information Technology, Vol. 6, Morishita Publishing, 1989 (in Japanese).

20. J.J. Koenderink, The structure of images, Biological Cybernetics, Vol. 50, 363-370, 1984.

21. A. Kumar, A.R. Tannenbaum, G.J. Balas, Optic flow: a curve evolution approach, IEEE Trans. Image Proc., Vol. 5, 598-610, 1996.

22. T. Lindeberg, Scale-space theory in computer vision, Kluwer, Boston, 1994.

23. A. Mitiche, P. Bouthemy, Computation and analysis of image motion: a synopsis of current problems and methods, Int. J. Comput. Vision, Vol. 19, 29-55, 1996.

24. H.H. Nagel, W. Enkelmann, An investigation of smoothness constraints for the estimation of displacement vector fields from images sequences, IEEE Trans. Pattern Anal. Mach. Intell., Vol. 8, 565-593, 1986.

25. M. Proesmans, L. Van Gool, E. Pauwels, A. Oosterlinck, Determination of optical flow and its discontinuities using non-linear diffusion, J.-O. Eklundh (Ed.), Computer vision - ECCV '94, Lecture Notes in Computer Science, Vol. 801, Springer, Berlin, 295-304, 1994.

26. E. Radmoser, O. Scherzer, J. Weickert, Scale-space properties of regularization methods, this volume.

27. C. Schnörr, Segmentation of visual motion by minimizing convex non-quadratic functionals, Proc. 12th Int. Conf. Pattern Recognition (ICPR 12, Jerusalem, Oct. 9-13, 1994), Vol. A, IEEE Computer Society Press, Los Alamitos, 661-663, 1994.

28. J. Shah, A nonlinear diffusion model for discontinuous disparity and half-occlusions in stereo, Proc. IEEE Comp. Soc. Conf. Computer Vision and Pattern Recognition (CVPR '93, New York, June 15-17, 1993), IEEE Computer Society Press, Los Alamitos, 34-40, 1993.

29. J. Sporring, M. Nielsen, L. Florack, P. Johansen (Eds.), Gaussian scale-space theory, Kluwer, Dordrecht, 1997.

30. J. Weickert, Theoretical foundations of anisotropic diffusion in image processing, Computing, Suppl. 11, 221-236, 1996.

31. J. Weickert, Anisotropic diffusion in image processing, Teubner, Stuttgart, 1998.

32. J. Weickert, On discontinuity-preserving optic flow, S. Orphanoudakis, P. Trahanias, J. Crowley, N. Katevas (Eds.), Proc. Computer Vision and Mobile Robotics Workshop (CVMR '98, Santorini, Sept. 17-18, 1998), 115-122, 1998.

33. J. Weickert, S. Ishikawa, A. Imiya, Linear scale-space has first been proposed in Japan, J. Math. Imag. Vision, Vol. 10, 237-252, 1999. 